\title{
Complex, multifocal, individual-specific attention-related cortical functional circuits
}

\author{
LUIS F. H. BASILE ${ }^{a, b}$
}

${ }^{a}$ Laboratory of Psychophysiology, Faculdade de Psicologia e Fonoaudiologia, UMESP Division of Functional Neurosurgery, Institute of Psychiatry

${ }^{\mathrm{b}}$ University of São Paulo Medical School, Brazil

\begin{abstract}
Recent studies focusing on the analysis of individual patterns of non-sensory-motor CNS activity may significantly alter our view of CNS functional mapping. We have recently provided evidence for highly variable attention-related Slow Potential (SP) generating cortical areas across individuals (Basile et al., 2003, 2006). In this work, we present new evidence, searching for other physiological indexes of attention by a new use of a well established method, for individual-specific sets of cortical areas active during expecting attention.

We applied latency corrected peak averaging to oscillatory bursts, from 124-channel EEG recordings, and modeled their generators by current density reconstruction. We first computed event-related total power, and averaging was based on individual patterns of narrow task-induced band-power. This method is sensitive to activity out of synchrony with stimuli, and may detect task-related changes missed by regular Event-Related Potential (ERP) averaging. We additionally analyzed overall inter-electrode phase-coherence.

The main results were (1) the detection of two bands of attention-induced beta range oscillations (around 25 and $21 \mathrm{~Hz}$ ), whose scalp topography and current density cortical distribution were complex multi-focal, and highly variable across subjects, including prefrontal and posterior cortical areas. Most important, however, was the observation that (2) the generators of task-induced oscillations are largely the same individualspecific sets of cortical areas active during the resting, baseline state. We concluded that attention-related electrical cortical activity is highly individual-specific (significantly different from sensory-related visual evoked potentials or delta and theta induced band-power), and to a great extent already established during mere wakefulness.

We discuss the critical implications of those results, in combination with other studies presenting individual data, to functional mapping: the need to abandon group averaging of task-related cortical activity and to revise studies on group averaged data, since the assumption of universal function to each cortical area appears deeply challenged. Clinical implications regard the interpretation of focal lesion consequences, functional reorganization, and neurosurgical planning.
\end{abstract}

Key terms: Attention, cortical electrical activity, high-resolution electroencephalography, slow potentials, source localization, functional mapping.

\section{INTRODUCTION}

Studies that aim at localizing the physiological changes that follow passive sensory stimulation or simple movement execution in cortical areas of individual human subjects, generally lead to uncontroversial, expected results. Thus, for instance, Electroencephalography and Magnetoencephalography (EEG and MEG) in such cases of highly focal sensory evoked potentials and fields can distinguish even the sources corresponding to stimulation of separate sections of the visual field or to tactile stimulation of different fingers. This precision may for instance be used for protection of sensorymotor areas during surgical interventions, when edema prevents visualization of the central sulcus (Wheless et al., 2004).

Address Correspondence to: L.F.H. Basile, M.D., PhD., Division of Functional Neurosurgery, Institute of Psychiatry, University of São Paulo Medical School, Av. Dr. Ovidio Pires de Campos 785, P.O.Box 3671, São Paulo, SP, 05403-010, BRAZIL, Phones: 55-11-30697284, 55-11-32846821 FAX: 55-11-2894815, e-mail: 1basile@usp.br 
Metabolic tracing studies, particularly fMRI, also allow the analysis of single subject sensory-motor data, and such paradigms are commonly used for testing and validation of the programs of analysis themselves. As opposed to this situation, a very different panorama arises from studies that directly interest Psychophysiology or Psychiatry, that is, those using paradigms including experimental tasks that involve any psychological variable beyond sensation and simple movement, from simple behavioral decisions, comparison of stimuli, expecting attention, memory or emotion. In spite of the great efforts to functionally map non-primary cortical areas (whose activity supposedly should correspond to various 'cognitive functions') and the resulting vast literature of the last decades, one can notice a clear lack of consensus across authors or laboratories. When one starts from the functional end, any unrestricted literature search regarding task-related cortical activity to any given psychological variable (e.g., memory evocation) will include changes, across studies, in most if not all cortical nonprimary areas. The same is true if one performs the literature search restricted to a given architectonic area, which will result in studies claiming a number of functions impossible to reconcile with each other.

Almost all studies on task-related cortical activity present data collapsed across subjects (group or grand averages), projected over an abstract, 'average brain'. Their obvious limitations include the anatomical individual variability in the structure of and, more critically, in the distribution of cytoarchitectonic areas onto gyri and sulci (Uylings et al., 2005). Recently, however, metabolic tracing studies emphasizing and presenting individual physiological and anatomical data are emerging, all of which demonstrate a large degree of interindividual variability in the encephalic distribution of task-related activity (e.g., Cohen et al., 1996; Herholz et al., 1996; Fink et al., 1997; Davis et al., 1998; Brannen et al., 2001; TzourioMazoyer et al., 2002), and in some cases, even to passive stimulation (Davis et al., 1998; Hudson, 2000). We do not refer here to any form of interindividual variability (such as exact coordinates of a center, or extension of physiological changes in one area; e.g. Vandenbroucke et al., 2004), but on the actual set of cortical areas active during a given task. Our own recent studies, following the conventional attempt to uncover universal areas across subjects, active during expecting attention to specific perceptual domains, have encountered this major shortcoming: First, by modeling the generators of a Slow Potential (SP; a class of event-related potentials, ERPs, which include the contingent negative variation, CNV) occurring in anticipation to taskperformance feedback stimuli, and expecting to find some pattern of prefrontal activity, we found a multifocal distribution of generators, both prefrontal and posterior cortical, although including two common prefrontal areas. But the actual set of areas was highly variable across subjects (Basile et al., 2002). Second, by comparing the SP generators corresponding to verbal, pictorial and spatial visual selective attention, such a complex and individually variable set of active areas was also found, and precluded any conclusion based on visual inspection: we developed an activity scoring method to allow statistical analysis but simultaneously preserving individual anatomical and physiological information (Basile et al., 2003). Since the task then used was complex, and included memorization and comparison of stimuli, the commonly claimed variability in task execution strategies could not be ruled out as a source of interindividual variability. We thus developed a task to allow the recording of SPs during (temporal) attention to simple visual stimuli, by simplifying the paradigm of spatial attention cueing of Posner (1980; Posner et al., 1980). However, the same high degree of interindividual variability in cortical activity distribution was once more observed (Basile et al., 2006).

Taken together, those studies on taskrelated cortical activity that preserve individual data, indicate that interindividual variability appears to be an inherent aspect of the normal functioning of the central nervous system. An interesting theoretical concept of biological "degeneracy" (Edelman and Gally, 2001), when applied 
to task performance and recovery of function (Noppeney et al., 2004), explicitly accounts for the inexistence of a simple, one-to-one isomorphic mapping between function and structure. We believe that the interindividual variability in sets of cortical areas engaged in any given task can account for most of the lack of consensus in cortical psychophysiology, since it is mainly based on group data averaging, which may emphasize areas that are strongly active in few subjects, and deemphasize areas which could be common to many subjects, but weakly active.

In this work, we present results from the search of some new electrophysiological correlate of expecting attention. We investigated more thoroughly the same simple task and subjects for whom SPs were analyzed (Basile et al., 2006). Most research on the electrophysiology of human attention has centered on the endogenous potentials of the P300 class, and on the modulation of sensory evoked potentials. However, such event-related potentials (ERPs) and their modulations may best be described as correlates of stimulus detection, and are only enhanced by expecting attention as an antecedent condition. So far, SPs are rigorously the direct correlates of attention. ERPs suffer from the limitation of time-locking to stimuli, and if one allows, in principle, for the occurrence of oscillatory activity bursts with no fixed relation to task events, they would thus be canceled out in regular ERPs. It is exactly this limitation of ERPs that has brought an increasing interest in studying electrical power measures that are not synchronized to stimuli, variously named as induced band-power (IBP), or Event-Related De- or Synchronization (ERD, ERS) for decreases and increases, respectively (Klimesh et al., 2000; Pfurtscheller, 2001). We here used a systematic IBP analysis to guide the averaging of oscillatory activity in narrow frequency bands, by a new application of a well known extension of stimulus-locked averaging: peak or corrected latency averaging. We first planned to compute task-induced EEG power (which lacks precise time and phase-locking to stimuli), and if some oscillatory activity were observed to increase during the pre-S2 time window, where SPs are maximal in amplitude, we would compute corrected latency averages (centered on peak amplitude of bursts instead of stimulus onset). Then, we intended to focus on the topographic and cortical generator analysis by current density reconstruction constrained by individual MRI data, such as we established for SP analysis, but still searching for a correlate of attention which would originate from a simpler, universal set of cortical areas across subjects. In particular among all frequency bands, there is a return of interest in theta $(3-7 \mathrm{~Hz})$ oscillations as a putative correlate of expecting attention. Theta rhythm may be observed in the ongoing EEG of waking, healthy individuals during tasks such as overt calculation with pen and paper (Mizuki et al., 1980), but in a small proportion of subjects (Takahashi et al., 1997). Although it has also been reported in cue-target (S1-S2) paradigm similar to the ones used for recording SPs (Nakashima and Sato, 1993), they could be alternatively accounted for by sustained mental effort, the uncontrolled stimulus presentation or overt movement.

\section{METHODS}

\section{Subjects}

Twelve healthy individuals with normal vision and hearing, 9 male and 3 female, participated in the study. They ranged in age between 20 and 45 years, with no history of drug or alcohol abuse, and no current drug treatment. Eleven subjects were current or former medical students. All subjects signed consent forms approved by the Ethics Committee of the University of São Paulo Hospital.

\section{Stimuli and Task}

A commercial computer program (Stim, Neurosoft Inc.) controlled all aspects of the task. Visual stimuli composing the cue- 
target pairs (S1-S2) consisted in small rectangles (eccentricity $\pm 0.8^{\circ}, \mathrm{S} 1: 100 \mathrm{~ms}$ duration, S2: $17 \mathrm{~ms}$ ). In half of the trials, the $\mathrm{S} 2$ rectangle contained a grey circle the task target - with $\pm 0.3^{\circ}$ of eccentricity. S1 was followed by S2, with onsets separated in time by 1.6 seconds. We instructed the subjects that a rectangle would be presented to indicate that 1.6 seconds later it would flash again but quickly, containing or not the target circle. The subject decided whether there was a target inside the $\mathrm{S} 2$ rectangle, and indicated presence of target by pressing the right button with the right thumb or absence of target by pressing the left button with the left thumb. We explicitly deemphasized reaction time in the instructions and measured performance exclusively by the percent correct trials, from the total of 96 trials comprising the experiment. An eye fixation dot was continually present on the center of the screen, as well as a stimulusmasking background, to prevent afterimages. To confirm the expecting attention index role of some possibly found EEG rhythm, we also used a passive stimulation control condition (S2s never contained targets), during which subjects were only required to fixate and relax.

\section{EEG Recording and acquisition of MRIs}

We used a fast $\mathrm{Ag} / \mathrm{AgCl}$ electrode positioning system consisting of an extended 10-20 system, in a 124-channel montage (Quik-Cap, Neuromedical Supplies,), and an impedance-reducing gel which eliminated the need for skin abrasion (Quick-Gel, Neuromedical Supplies,). Impedances usually remained below $3 \mathrm{kOhms}$, and channels that did not reach those levels were eliminated from the analysis. To know the actual scalp sampling or distribution of electrodes in each individual with respect to the nervous system, we used a digitizer (Polhemus,) to record actual electrode positions with respect to each subject's fiduciary points: nasion and preauricular points. After co-registration with individual MRIs, the recorded coordinates were used for realistic $3 \mathrm{D}$ mapping onto MRI segmented skin models, and later used to set up the source reconstruction equations (distances between each electrode and and each dipole supporting point). Two bipolar channels, out of the 124-channels in the montage were used for recording both horizontal (HEOG) and vertical electrooculograms (VEOG). Left mastoid served as reference only for data collection (common average reference was used for source modeling) and Afz was the ground. We used four 32-channel DC amplifiers (Synamps, Neuroscan Inc.) for data collection and the Scan 4.3 software package (Neurosoft Inc.) for initial data processing (until computation of averages). The filter settings for acquisition were from DC to 30 $\mathrm{Hz}$, and the digitization rate was $250 \mathrm{~Hz}$. Thus, the gamma band was not collected, due to concerns with noise from the laboratory environment and absence of Faraday shielding. The EEG was collected continuously, and epochs for averaging spanned the interval from $700 \mathrm{~ms}$ before S1 to $400 \mathrm{~ms}$ after S2 presentation. Baseline was defined as the $400 \mathrm{~ms}$ preceding S1. Artifact elimination was automatic: epochs containing signals in either HEOG or VEOG channels above +50 or below $-50 \mathrm{mV}$ were eliminated. In our montage, the VEOG detected blinks as deflections above $130 \mathrm{mV}$ in the positive direction.

MRIs were obtained by a 1.5 Tesla GE machine, model Horizon LX. Image sets consisted in 124 T1-weighed sagittal images of 256 by 256 pixels, spaced by $1.5 \mathrm{~mm}$. Acquisition parameters were: standard echo sequence, 3D, fast SPGE, two excitations, $\mathrm{RT}=6.6 \mathrm{~ms}, \mathrm{ET}=1.6 \mathrm{~ms}$, flip angle of 15 degrees, F.O.V = $26 \times 26 \mathrm{~cm}$. Total acquisition time was around 8 minutes.

\section{Frequency-Time analysis (Task-induced BandPower) and Power Scalp Topography}

After artifact rejection, the signal from each channel was spectrally analyzed by means of a Short Time Fourier Transform (STFT), to obtain frequency-time charts of the induced (stimulus related, but not stimulus-locked) and evoked (stimulus-locked) spectrum of the interval from $700 \mathrm{~ms}$ previous to $\mathrm{S} 1$, to $400 \mathrm{~ms}$ after S2. To obtain the induced power spectrum (Tallon-Braudry; 1996), the 
time-frequency decomposition was made for each electrode and each trial, from DC to 30 $\mathrm{Hz}$, and the resulting charts were then averaged. The evoked power spectrum was obtained applying the spectral decomposition to the averaged signal. Recently, it has been demonstrated that this method is mathematically equivalent to others like the Hilbert transform, or wavelet decomposition, and that each of them yields equivalent results in practical applications to neuronal signals (Bruns and Eckhorn, 2004). The decomposition was computed on the EEG tapered by a sliding Hamming window, 256 points in size for frequencies over $5 \mathrm{~Hz}$, and 512 points between 2 and $5 \mathrm{~Hz}$, with a temporal resolution of $\mathrm{N} / 10$ ( $\mathrm{N}$ being the number of temporal points of the raw signal), and a frequency resolution of 4 bins per Hertz. Then, we normalized the average power for each electrode to obtain Z-scores of increments or decrements in each frequency bin with respect to the power in the same frequency during the baseline $\left(<\mathrm{P}_{\mathrm{j}}>\right.$ $=\left(\mathrm{P}_{\mathrm{j}}-\mu_{\mathrm{j}}\right) / \sigma_{\mathrm{j}}$; given $\mathrm{P}_{\mathrm{j}}=$ spectral power at each time point in electrode $\mathrm{j}$ ), $\mu_{\mathrm{j}}$ and $\sigma_{\mathrm{j}}$ are the mean and standard deviation, respectively, of the average power during the baseline at this electrode).

We computed realistic three-dimensional topographic maps of the scalp distribution of normalized power, at each frequency band that demonstrated task-induced changes, for each subject, over the reconstructed scalp anatomy. To this purpose, we used a commercial sotfware (Curry V 4.6, Neurosoft Inc.), which co-registered individual MRI sets (skin model, see below), with the actual position of each electrode with respect to common landmarks, and linearly interpolated the instantaneous values of power to obtain continuous maps.

\section{Computation of corrected latency burst averages}

According to the observed induced frequency bands and time windows where they occurred for each individual, the original artifact-free EEG epochs (ranging from $700 \mathrm{~ms}$ before S1 through $500 \mathrm{~ms}$ after S2) from each subject were filtered around the bands of interest (Butterworth, 96dB rolloff, typically 1-3 Hz for delta, 3-7 for theta, 7-9 for alpha1, 9-12 for alpha2, around $25 \mathrm{~Hz}$ for beta2, a wider range for beta1, and in some subjects for obtaining an additional low beta band, between 13 and $15 \mathrm{~Hz}$ ). The resulting filtered epochs were then subject to an algorithm developed by us to search for the peaks of bursts within the task-time windows of interest. Filtered epochs were thus cut again starting from positive voltage peaks, resulting in new epochs, ranging from $400 \mathrm{~ms}$ before to $400 \mathrm{~ms}$ after the peaks. A minimum of 60 epochs was averaged for each individual and frequency band, using each channel in the search for peaks. Each channel at a time thus served for peak detection, to create a multi-channel average, in which all remaining channels simply followed the latency correction of the leading channel. In this way, all systematic phase relations were preserved. Then, a grand average was computed using the averages obtained by guidance from each channel. Since this method would in principle suffer from the limitation of confounding any possible systematic time (direction) relations between active areas, for instance if groups of areas were active in sequence in a given frequency band, we also computed partial averages using groups of guiding channels ranked for latency of occurrence of peaks. That is, using only the first one fourth of channels (those with overall shorter peak latencies), and second, third and last fourth of channels. We also performed independent analyses to study possible time relations between groups of electrodes (next section). Finally, in all cases we also computed pre-S1 burst averages (representing the baseline topography for each frequency band), where the program searched peaks from -400 to $0 \mathrm{~ms}$ before $\mathrm{S} 1$, for comparison with the task-induced bursts.

\section{Inter-electrode phase-synchrony analysis}

Since a systematic and complete phase analysis of the present data would consist in a separate and major work, we decided to present only a first approach: we computed only the overall pattern of phase relations, in the form of averages across all pairs of channels. This suffices to answer the 
question about whether there are task-related phase changes in correspondence to induced power. The practice of separately computing phase is becoming common in event-related power studies (Fell et al., 2004; Hanslmayr et al., 2006). Due to volume-conduction effects, we selected a group of 25 regularly interspaced electrodes from the original array to compute this index. A similar procedure used to obtain the power spectrum of the signal was used to compute the phaselocking value between electrodes (Lachaux et al., 1999). That is, a STFT of the signal from each electrode and trial was computed to obtain the instantaneous angular phase for each frequency, during a time window centered at time $t$. Then, after subtracting the constant angular phase, a complex vector of unitary value was constructed for each channel, trial, frequency and time. With this value, a matrix of differences of phase values between electrodes, in each trial, was computed for each frequency-time, and then averaged over all the trials. Using the modulus of this complex value, we obtained for each pair of electrodes, in each frequency and time point, a phase-difference value between 0 (random phase relation) and 1 (constant phase relation). That is, $\Phi \mathrm{i}(\mathrm{f}, \mathrm{t}, \mathrm{k})$ being the phase value of electrode $i$, at frequency $\mathrm{f}$, time $\mathrm{t}$, and trial $\mathrm{k}$, and $\Phi \mathrm{j}$ (f, t, $k$ ) the phase value of electrode $j$, in the same frequency, time and trial, the phase-locking value was computed as

$$
\Phi \mathrm{ij}(\mathrm{f}, \mathrm{t})=1 / \mathrm{N} \text { I } \Sigma \mathrm{k}=1 \mathrm{~N} \Phi \mathrm{i}-\Phi \mathrm{j} \mathrm{l} .
$$

The phase-locking values obtained for the time interval posterior to stimulus presentation was then Z-normalized by the values obtained during the baseline interval in the same way as time-frequency spectral matrices. Finally, we verified whether there were statistically significant correlations between task-related power and phase, in frequency bands where we could visualize any such systematic relations.

\section{Intracranial source reconstruction}

The computed averaged bursts, MRI sets and electrode position digitization files were the raw data for all further analysis (Curry V 4.6, Neurosoft Inc.). A detailed description of the reconstruction procedure, and a discussion on the criteria for method choice and shortcomings, as well as on critical steps, may be found in the methods section of our previous publications (Basile et al., 2002; 2006). Noise in the data was defined as the variance of the $20 \%$ lowest amplitude points in each average. For the inclusion of a 'noise component' into the source model, the physical unit-free or 'standardized' data (with retained polarity) were decomposed by Independent Component Analysis (ICA), which searches for the highest possible statistical independence or redundancy reduction between components (in this case, space-time averaged data patterns), a robust method of blind signal decomposition/ deconvolution (for a review see, e.g. Hyvarinen and Oja, 2000). ICA was applied to each individual's whole space-time data set, i.e., to the $\mathrm{m} \times \mathrm{n}$ data matrix (m used channels times 201 time samples corresponding to the $800 \mathrm{~ms}$ composing the averaged bursts). Finally, we fed the reconstruction algorithm with the main ICA component(s) as data to be fitted. Thus, the 'noise component' of the model was defined as the sum of remaining components (with loadings below $\mathrm{SNR}=1$ ), all of which added together lead invariably to negligible scalp potentials when compared to the main components. In practically all cases, only two space-time ICA components were then modeled. MRI sets were linearly interpolated to create 3-dimensional images, and semiautomatic algorithms based on pixel intensity bands served to reconstruct the various tissues of interest. A Boundary Element Model (BEM) of the head compartments was implemented, by triangulation of collections of points supported by the skin, skull and cerebrospinal fluid (internal skull) surfaces. Mean triangle edge lengths for the BEM surfaces were, respectively, 10, 9 and $7 \mathrm{~mm}$. Fixed conductivities were attributed to the regions enclosed by those surfaces, respectively, $0.33,0.0042$ and $0.33 \mathrm{~S} / \mathrm{m}$. Finally, a reconstructed brain surface, with mean triangle side of $3 \mathrm{~mm}$, served as the model for dipole positions, corresponding to 
a minimum of 20 thousand points. The electrode positions were projected onto the skin's surface following the normal lines to the skin. The detailed description of the assumptions and methods used by the "Curry 4.6" software for MRI processing and source reconstruction may be found elsewhere (Curry 4.0 User Guide, 1999; or e.g., Buchner et al., 1997; Fuchs et al., 1998; Fuchs et al., 1999). The analysis program then calculated the lead field matrix that represents the coefficients of the set of equations which translate the data space (SNR values in the set of channels per time point) into the model space (above 20 thousand dipole supporting points). The source reconstruction method itself was Lp norm minimization, with $\mathrm{p}=1.2$ both for data and model terms. The regularization factor, or $\lambda$ values to be used, which typically converged after repeating the fitting process three to four times ( $\lambda$ gives the balance between goodness of fit and model size). Resulting foci of current density were inspected with respect to the individual anatomy directly, a straightforward procedure allowed by our software, in terms of which estimated cytoarchitectonic areas they covered (and scored for relative intensity; Basile et al., 2003). The Brodmann areas containing current foci were tabulated after verification by comparison with classical illustrations and the conventional Talairach and Tournoux atlases (1993, 1997).

\section{RESULTS}

\section{Task Performance}

All subjects reported that performance was relatively easy, given that effort of attention to the S1-S2 pairs was made. The overall average performance was $88.5 \%$ correct responses (standard deviation $8.3 \%$ ).

Temporal and topographic characteristics of task-Induced power and Inter-electrode phase analysis

We first describe the overall findings, common to all subjects, regarding the behavior of each induced power band with respect to task events, and their scalp topography. Task-induced theta power was not present in the ISI interval, showing a clear post-stimulus increase pattern in all subjects, practically returning to baseline level during the ISI. The two peaks corresponded to around $180 \mathrm{~ms}$ post $\mathrm{S} 1$ or $\mathrm{S} 2$, thus coinciding with the latency of the N200 evoked potential component. The same purely stimulus dependent behavior was observed for induced delta power, but in this case the peaks occurred later, around $350 \mathrm{~ms}$, and in almost all subjects with much higher amplitude after S2 (suggesting at least partial relation with the P300 component, due to the latency and more task-relevant S2). Figure 1(1) shows the overall task-time behavior of the induced power, with data collapsed across electrodes and subjects. Induced theta presented an overall increase in peak amplitude with respect to baseline of $13.8 \%$ $( \pm 11.5 \%)$, but ranging from no change in two subjects (increases around 6\% in four subjects), to $34 \%$. Delta presented an overall increase of $39.2 \%( \pm 42.3 \%)$, but ranging from reduction in two subjects (to 62 and $91 \%$ from baseline), virtually no change in one subject, to $89 \%$. Regarding the scalp topography of delta and theta, both bands had a clearly posterior distribution of task-induced power maxima, typically with a double occipital peak, in all cases either or both power peak distributions being coincident with the N200 peak voltage distribution. Figure 2 shows the induced power and corresponding topography of all bands (at peaks of task-induced power increases), grand average across subjects, collapsed across the montage and projected on median head size individual. The time pattern of induced power was virtually identical to the evoked pattern for theta and delta bands. A more critical finding, which we will resume in the discussion in connection with other general results (valid for all frequency bands), was the identical topography of evoked or induced power. Alpha desynchronization is not clearly seen possibly due to the collapsing across electrodes for time-frequency plot computation. 
(1)

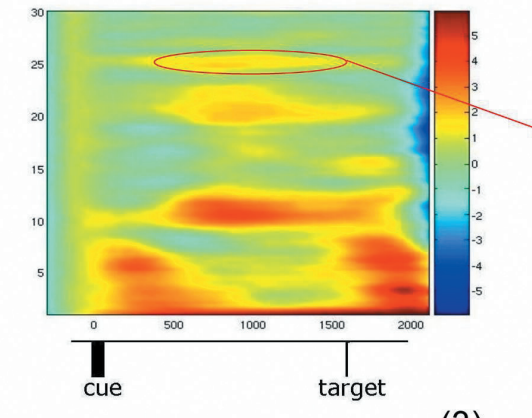

(2)

(3)
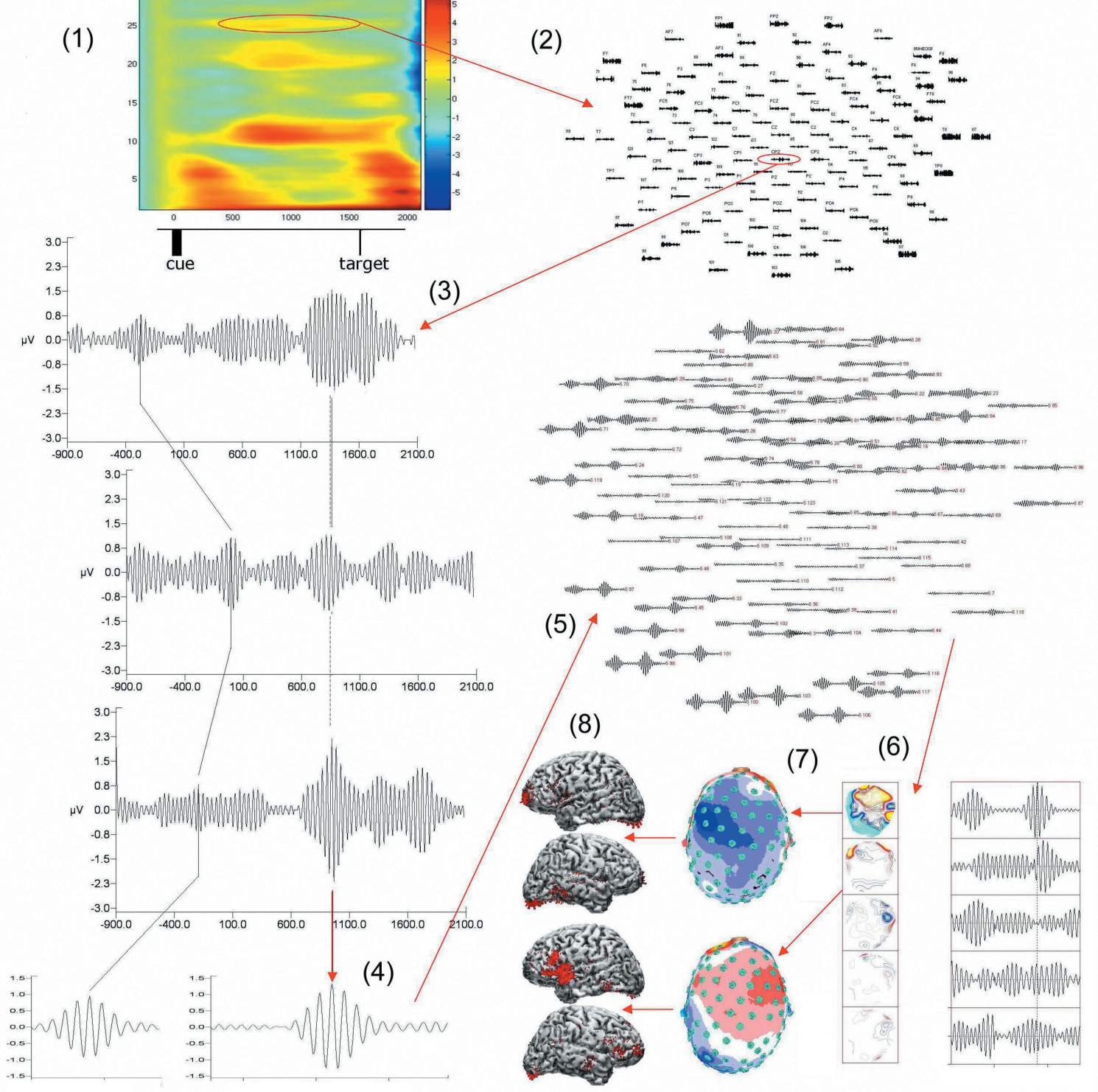

(5)

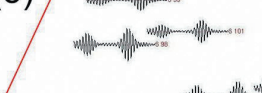

(8)

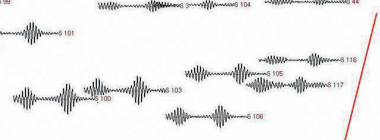

(7)

(6)

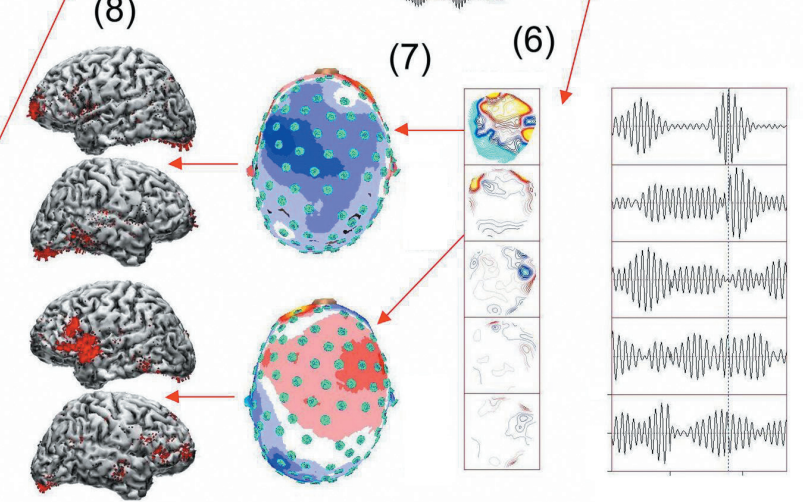

Figure 1: Schematic representation of the main steps of the method: (1) Computation of taskinduced band-power. In this figure, real data is presented, collapsed across channels and subjects (numbers indicate $\mathrm{z}$-score - power relative to baseline). One may appreciate the overall time course of task-induced power changes, which were fairly common across subjects (see text for few frequency bands where exceptions occur, low beta and alpha-1). (2) EEG Epochs were filtered in individual-specific narrow frequency bands. (3) Based on each channel, positive voltage peaks were automatically detected in time windows of power increases, and (4) multi-channel latency corrected averages computed. (5) A final multi-channel average was computed, between multi-channel averages guided by each channel. (6) Independent Component Analysis (ICA) separated the (7) main and second space-time components to feed the (8) Current Density Reconstruction (CDR) algorithm. 


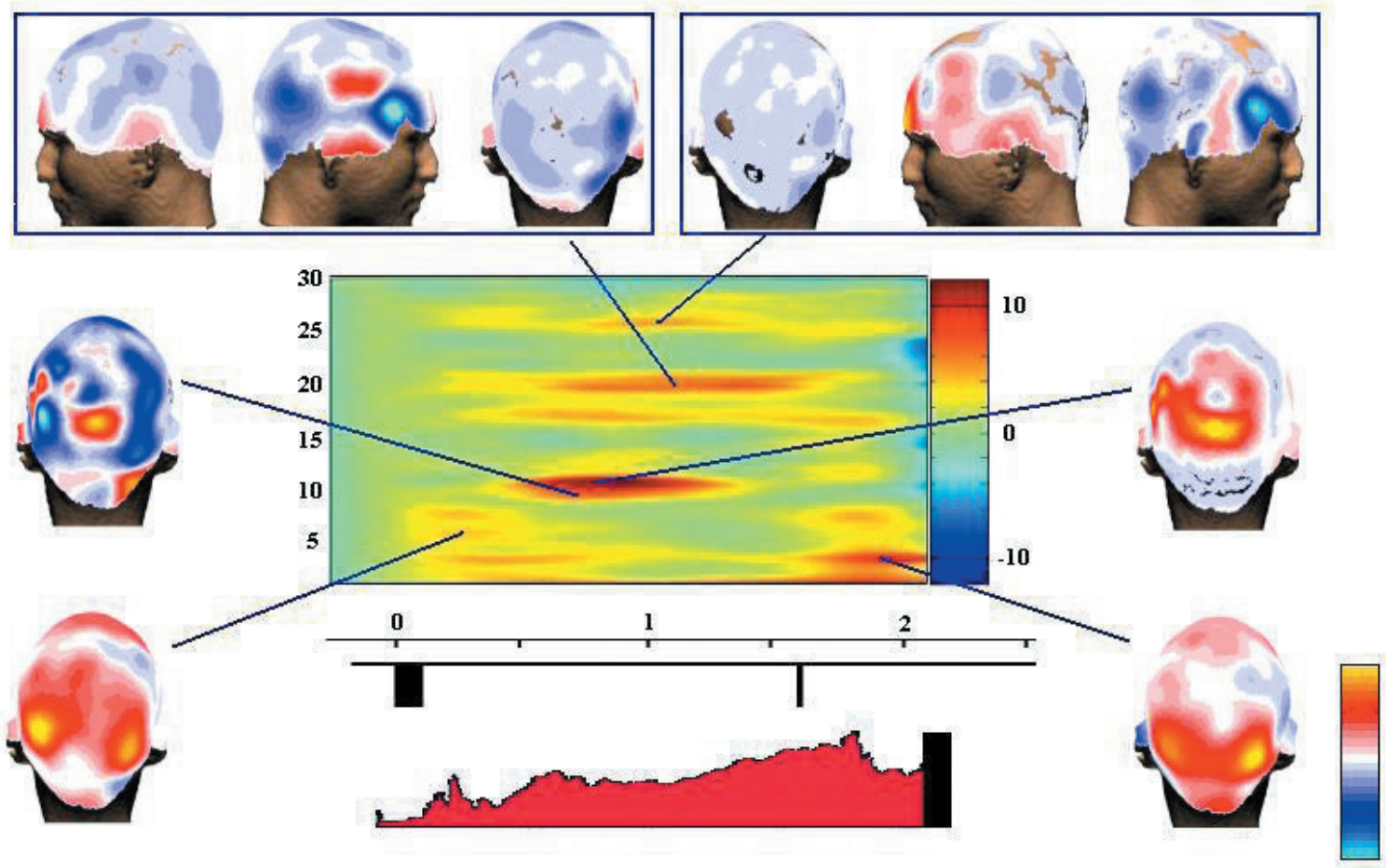

Figure 2: Task-induced band-power of one example individual, collapsed across all channels, and corresponding topographic distribution of the main points of change, that were common to all subjects. Color scale: extreme of power changes (yellow and magenta) correspond to z-score equal to 9.8 standard deviations, 'hot' colors indicate increase and 'cold' decrease relative to baseline. Below, time course of stimuli and mean event-related potential global field power (bar $5 \mu \mathrm{V})$.

The induced alpha range, on its turn, presented a complex multiphasic pattern during the task time, with an inflection showing a relative decrease in power around $200 \mathrm{~ms}$ after the stimuli (alpha desynchronization), another opposite inflection reflecting a small increase around $400 \mathrm{~ms}$ after stimuli (which is the latency of the peak of evoked alpha and delta power), and a major increase peaking roughly around $700 \mathrm{~ms}$. Induced alpha oscillations were increased in all subjects, ranging from 2 to $57 \%$ from baseline (overall $30,4 \% \pm 16,3 \%$ ). The scalp topography of induced alpha power also showed the expected posterior distribution of highest power. However, the actual isocontour map shapes were different from the simple pattern resembling the N200 evoked potential component that we observed for theta and delta ranges (and also with no trivial relation to the P300 topography, which appear to combine mainly delta, theta and alpha frequencies; see e.g., Cacace and McFarland, 2003). Each individual presented a fairly complex and specific map shape. Since for alpha, evoked activity occurred exactly where the least of total induced power was observed ('post-stimulus' time window), it served as the best example for comparison between topographies of the two methods of computation: in this case also, the topographic rendering of the data resulted, in all subjects, in identical evoked and induced maps. Another interesting finding, which was also previously observed in our laboratory, was the clear presence of evoked alpha throughout the ISI (peaking in the ISI with an overall $71 \%$ of the maximum post-stimulus, evoked alpha). Given the long ISI (1.6 seconds) with respect to alpha wavelength, it is curious that so many alpha cycles could be synchronized with the task events. That is, in no other frequency band do we see such lasting synchronization. 
We may here summarize the results of phase analysis, given that the proposed correlative analysis was applied only to the beta range, discussed below. In all frequency bands but beta, the task-related phase coherence changes were complex and variable across subjects, to various degrees, depending on the sub-band in consideration. In all such variable cases, except for delta, a computation of phase coherence collapsed across subjects lead to virtually flat waveforms. For delta, however, at least one common aspect was retained in the group average: a peak of inter-electrode coherence increase at 450 $\mathrm{ms}$ (of around $\mathrm{z}$-score $=0.6$ ), and an equally low amplitude decrease during the ISI, peaking at $1200 \mathrm{~ms}$. To our surprise, given the highly regular induced-power pattern in time across subjects, theta inter-electrode coherence was the most variable. Subjects even presented opposite results during the peaks of post-stimulus power, with only three subjects presenting parallel increases in power and phase coherence, and three other presenting increases only during the ISI. Alpha 1 was the most variable in time patterns of coherence changes. For alpha 2, five subjects presented overall coherence reduction during the period of increased power, in the center of the ISI, where three subjects presented increases. The beta range, however, presented a relatively simpler, more common aspect across subjects, in the form of changes in coherence roughly parallel with power. We thus proceeded to analyze intra-individual correlations between the two variables, and the results supported the visual impression: beta 1 had significant non-parametric correlations in 11 subjects, and beta 2 , highly significant correlations in 10 subjects. The implications of those findings will also be considered in connection with the other results to allow for a more panoramic view of oscillatory activity, in the discussion.

There were two new and main findings in this work. The first regarded the beta range. In all subjects, a narrow induced beta band around $25 \mathrm{~Hz}$ was observed, throughout the ISI, peaking during the preS2 time range, following a time pattern that we originally expected to fit a putative attention-related induced theta band. In addition, all subjects presented a broader beta band roughly around $21 \mathrm{~Hz}$, and some subjects another narrow band close to 15 $\mathrm{Hz}$, but more variable in frequency and time pattern. Given the task-time distribution of beta, mainly the $25 \mathrm{~Hz}$ band, and the vague but old association between beta activity and behavioral arousal (that impelled the widespread but still controversial betaenhancement by biofeedback; Ramirez et al., 2001), we immediately thought beta could fulfill our expectation of a new index of expecting attention-induced activity. When computing the scalp distribution of beta power, this suspicion increased, due to the qualitative similarity with our findings regarding SPs: The topography of induced beta power changes was complex, multifocal, including frontal and temporal, as well as more posterior peaks, and highly variable across subjects. Finally, there was a statistically significant increase in beta power during the ISI, when the task was compared to the passive stimulation control condition: beta mean global field power from 500 through $1600 \mathrm{~ms}$, differed significantly between conditions both in parametric paired samples t-test (for beta1, $\mathrm{p}=0,004$; beta $2, \mathrm{p}=0,012)$, as in nonparametric tests (Wilcoxon test, $\mathrm{p}=0,005$ for beta1 and $p=0,012$ for beta2; Sign test, $\mathrm{p}=0,006$ for beta 1 and $\mathrm{p}=0,039$ for beta 2 ). Figure 3 shows beta mean global field power collapsed across individuals, and zscore of power scatter-plot in both conditions and bands. This also corroborated our idea of the role of induced-beta as an index of expecting attention. We thus focused on the source reconstruction of the task-induced beta bursts, presented in the next section.

\section{Source reconstruction}

The second new and important finding of this work was evident after computation of our corrected latency burst averages for source reconstruction: Since we computed burst averages for the pre-S1 baseline as well as for the task periods proper, in almost all cases (subjects and frequency 
bands), the topography of baseline activity was identical to the main component of task-induced oscillations. That is, it was clear that the same sources already active before stimuli composing the trials, presumably task-independent, were the main sources active during task execution. Moreover, very similar topography of baseline oscillations across frequency bands was observed within subjects. The only exception was the alpha band in half of the subjects, which had a peculiar topography, different and prevailing over the pattern similar across all remaining frequency bands, but that also remained the main alpha component during the task time window as well. It must be emphasized here that the topography observations were independent from amplitude of oscillations, whose task changes were variable across bands. Since those results were absolutely unexpected, we performed a comparison, using four subjects, between the averages computed for the pre-S1 baseline and
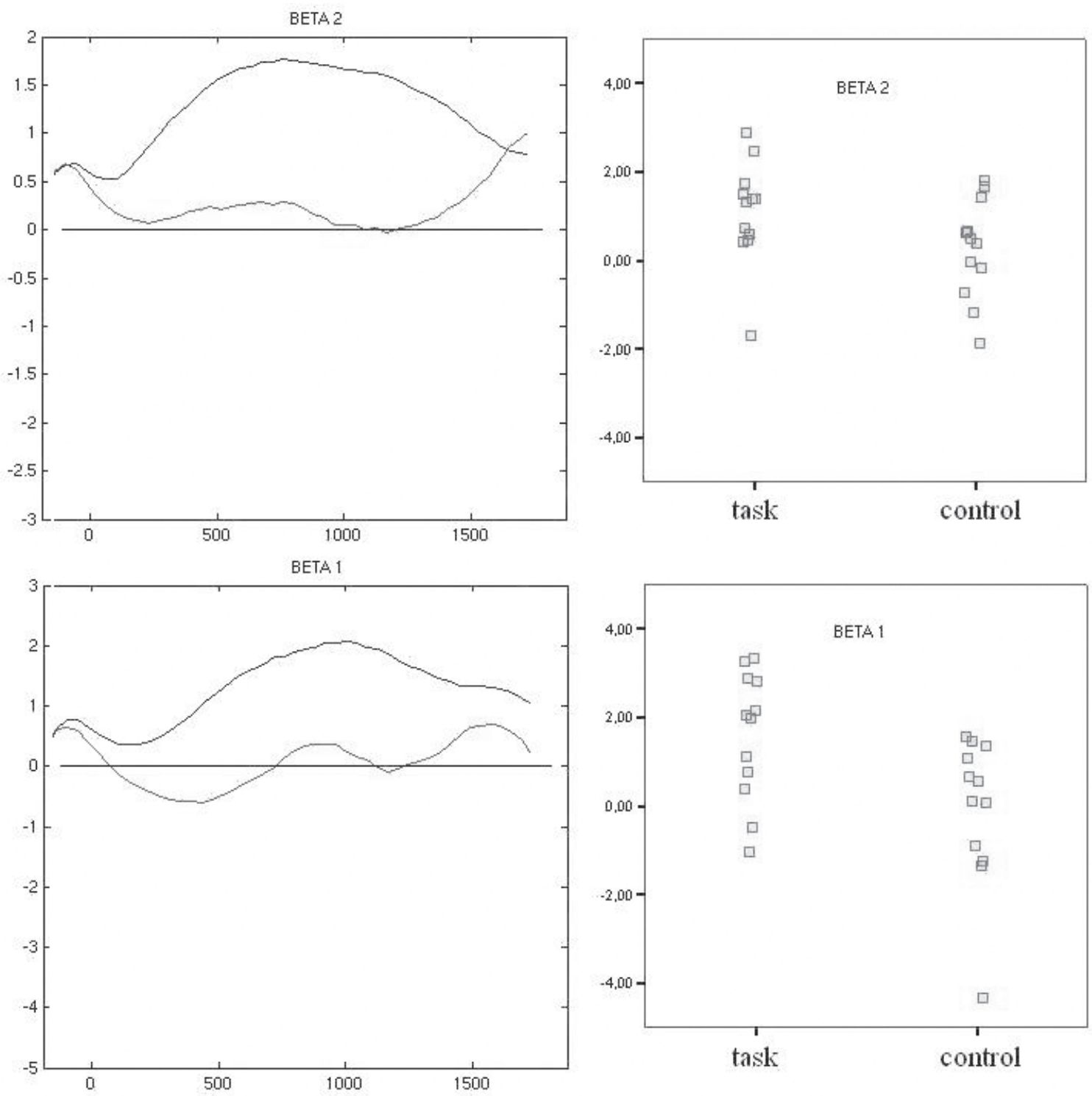

Figure 3: (left) Overall pattern of task-induced power increases in the beta range, collapsed across electrodes and individuals (upper curves), compared to passive stimulation control condition (bottom curves). (right) Individual induced beta power Z-score distribution in both conditions. 
similar averages computed for a resting condition of a few minutes which preceded the start of the experiment (filtered continuous EEG was marked in local voltage peaks using a refractory period of $800 \mathrm{~ms}$, to avoid overlap between epochs, since no task events were present). The same topography of baseline activity was thus observed, indicating that the pre-S1 baseline indeed reflects task-unrelated activity. Otherwise, it would be conceivable that baseline activity could still reflect task engagement, due to the cyclical nature of the task, i.e., if trial expectation were physiologically identical to relevant S2 expectation. In one subject, we also replicated the experiment after one month, and the same topography of baseline activity was observed. It is critical for future studies, however, that in three subjects who participated in previous experiments, four and six years before, we observed different topographies of resting condition oscillations, thus suggesting a spontaneous 'migration' of the mainly active areas in a given subject in this time range.

Corresponding to the complex scalp distribution of beta induced power, the source reconstruction results indicated the same complexity: Multifocal cortical current distribution, was highly variable across subjects, including frontal and posterior cortical sources in all subjects. As simple means of quantifying higher variability of beta topography, obvious to visual inspection (figure 4), when compared to evoked potentials or stimulus-related induced power (theta was chosen for comparison), we computed a deviation index. This type of index has proved successful in distinguishing the more complex and variable topography of SPs of a group of schizophrenia patients from healthy subjects (Basile et al., 2004). It is a quadratic norm or Euclidian distance between each individual's data set and the group averages collapsed on the montage (square root of electrode-by-electrode squared difference in voltage, divided by the number of channels): i.e., a scalar measure indicating individual distance from the norm, proportional to electrical power.
To emphasize topography and not to allow for absolute power to influence the measure, we first normalized beta and theta power across individuals into a common value. Results show clearly the larger dispersion of individuals from the (least representative) beta average, as compared to the dispersion from the group theta average (figure 4, where deviation (power) values are z-score normalized). The difference between beta and theta deviation indexes is statistically highly significant (Wilcoxon: $\mathrm{p}=0.002$; Sign test: $\mathrm{p}<0.001$ ). The partial averages computed from subsets of electrodes as explained in methods were identical to each other, and indicate absence of systematic sequential activation between beta generating areas. Added to the interelectrode phase analysis results, they suggest a tight phase synchrony between all such areas. Figure 5(a) shows the current distribution in each subject, accounting for the main ICA space-time data component, that is, the baseline activity component, which was enhanced (by $33.5 \%$ in average peak amplitude; $s t d=17.1 \%$; range $=6$ to $67 \%)$ and synchronized across channels during the ISI, pre-S2 period. Figure 5(b) shows the second component (exclusively or task-induced 'proper', i.e., not present during the pre-task period), of an overall relative intensity of $10.7 \%$ of the main component in electrical power ( $\mathrm{std}=12.2 \%$, ranging from less than $1 \%$ in one subject to $36 \%$ in two subjects, but within 3 to $13 \%$ in the remaining subjects), but in all cases of sufficient SNR for source reconstruction (average $\mathrm{SNR}=1.8$; $\mathrm{std}=0.7$; range $=1.05$ to 3.3). We may notice the individual-specific pattern of relative current distribution, especially conspicuous for the second, exclusively task-related component. Only parietal area 7 shows some level of activity in all subjects corresponding to the main, baseline component (although highly variable in intensity relative to maximum current). The reconstruction results for the beta-1 (around $21 \mathrm{~Hz}$ band) resulted in current distributions of main components identical to beta- 2 in all subjects. The scalp topography of the second component of beta- 1 burst averages, on its turn, in most of the subjects demonstrated a partial overlap 
with the second component of the $25 \mathrm{~Hz}$ band. Accordingly, reconstruction results showed very similar patterns in most cases, typically with the beta-1 set of current foci representing part of the set seen for beta-2. In some subjects, however, few additional (i.e., complementary to beta-2) weak sources were also observed.
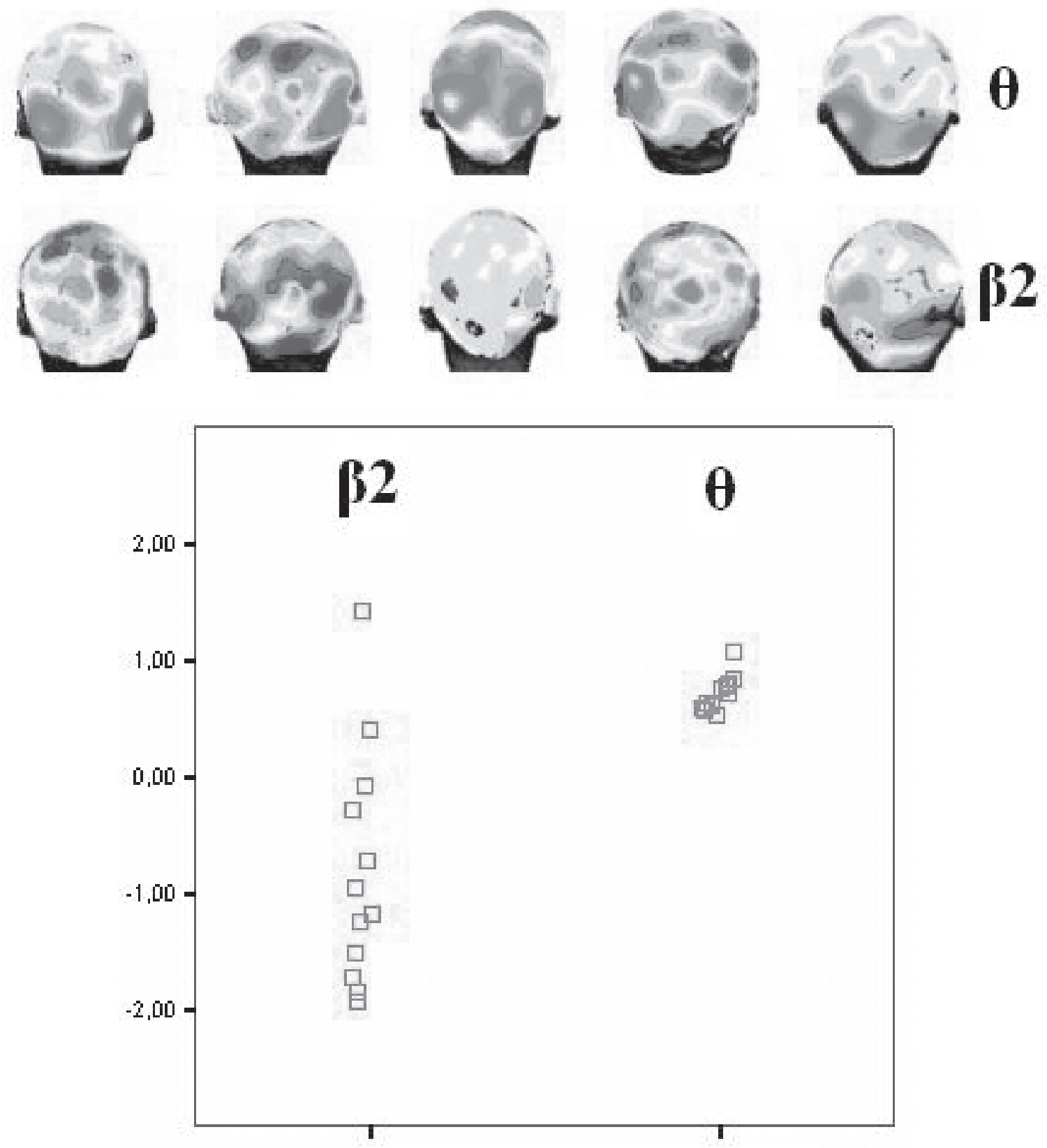

Figure 4: On top, examples of individuals presenting similar topography of task-induced theta activity, for whom beta distribution seen from the same angle is clearly more variable across subjects. Below, topographic deviation of each individual from normalized mean, between beta 2 and theta bands. Deviation was defined as the quadratic norm of the electrode-by-electrode difference between individual and group averaged data (across the montage, see text for details), as simple means to quantify the higher beta variability depicted by visual inspection. 

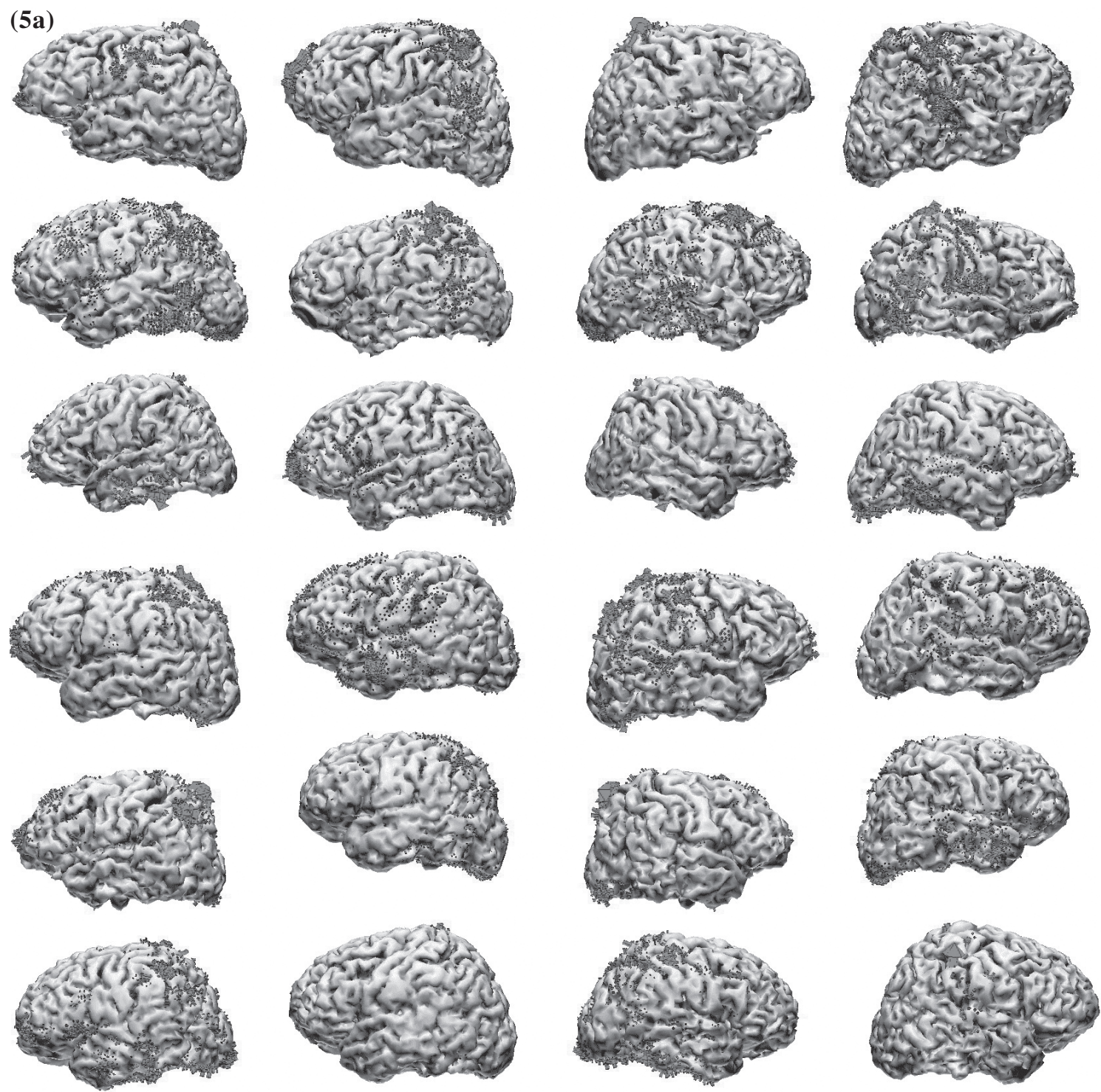

Figure 5: Current density reconstruction results for all subjects. Current density indicated by small red arrows, (arrow size proportional to local current density). (a) Main component, identical with main component obtained for baseline activity. (b, overleaf) Second, task-exclusive component. Numbers indicate relative power, in percentage, with respect to the main component from each individual, presented in the same order as in (a).

\section{DISCUSSION}

The first conclusion from this work regards the lack of theta power enhancement during the pre-S2 time window, where we would expect a direct correlate of expecting attention to have its maximum amplitude. Indeed, both theta and delta induced bandpower behaved clearly as post-stimulus phenomena, with peak amplitudes at posterior scalp regions, very similar in topography to the N200 component of the visual evoked potential. In all subjects, theta induced power peaked in coincidence with the peaks of the N200, and delta in the time window occupied by the P300 component. In ten subjects, the topography of theta power was practically coincident with the one from the N200 (typically double peaks at the occipital region); in the 
(5b)
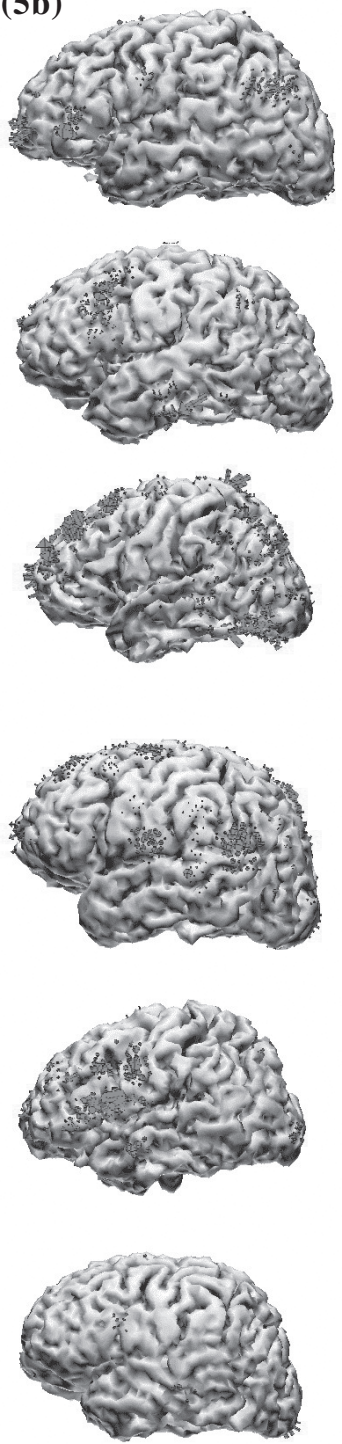
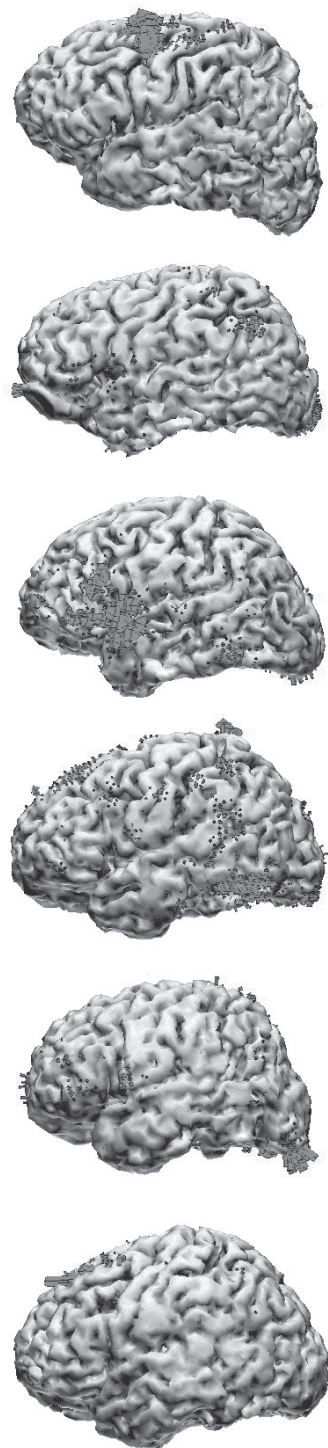
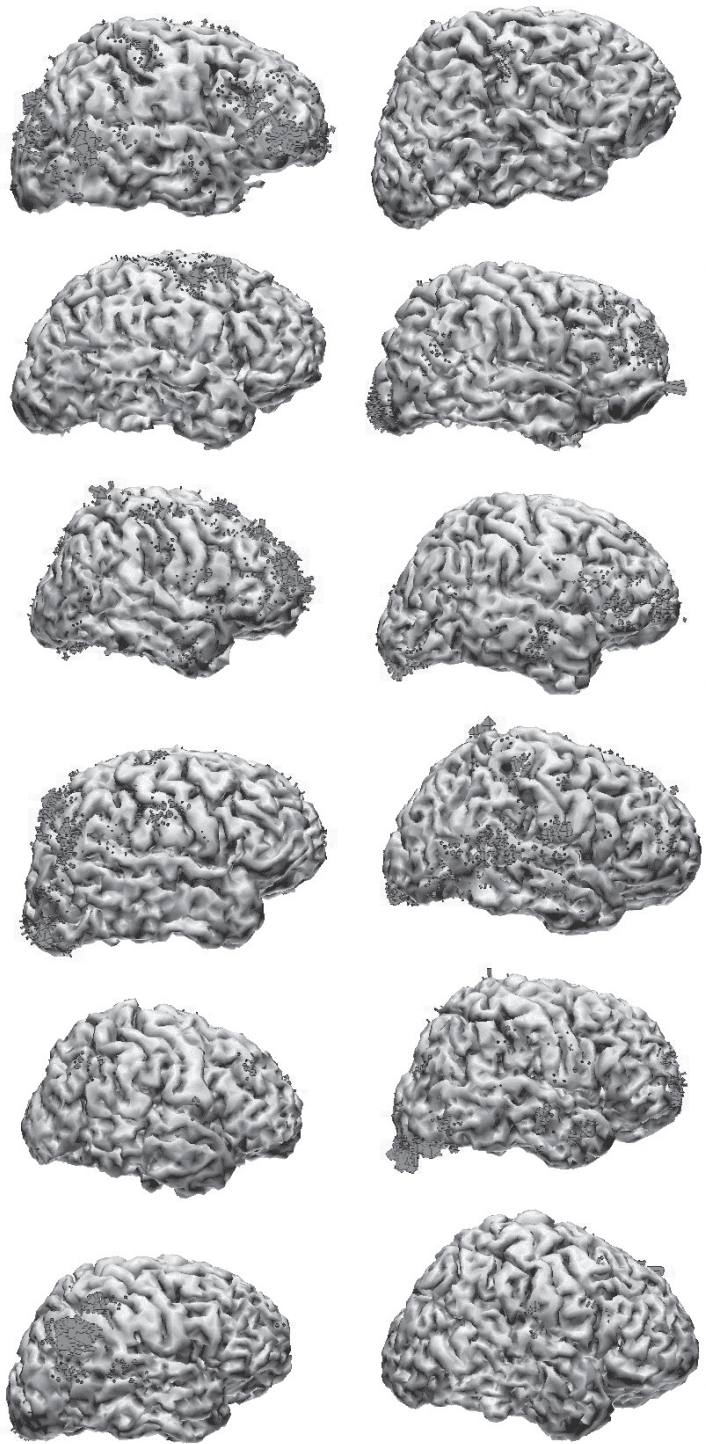

two remaining subjects, delta topography was closer to N200. Thus, both power bands appear simply to contribute to the composition of the evoked potential, and the method here used should not add much information with respect to the conventional ERP: induced and evoked power are coincident in this case, and the most important fact that seems to occur in these bands is a synchronization between their generators with respect to the stimuli, or phase resetting: In two subjects, delta burst averages were actually decreased in amplitude and unchanged in one, whereas theta maintained its amplitude in two subjects and showed very small increases in four subjects. This type of finding is commonly reported in the literature, and discussed below in conjunction with results valid for all bands.

With respect to the alpha range induced band(s), a thorough consideration would require an independent and major work due to complexity, the same being the case for a complete phase analysis. We thus discuss here only a few points which support some views under current discussion in the literature, especially our contribution that regards its topography. Half of the subjects presented a distinct alpha- 1 band - around 8 
$\mathrm{Hz}$ - in addition to the alpha-2 band centered around $11 \mathrm{~Hz}$, and the remaining half, exclusively a broad or narrow alpha- 2 band. Induced alpha behavior in relation to task time was multiphasic, with a relative (seen as an inflection) reduction/ desynchronization around $200 \mathrm{~ms}$ after the stimuli, a relative peak (almost unnoticeable when compared to the maximum induced alpha) overlapping with the delta peak and the P300 evoked potential, and maximum power at the center of the ISI, overall around $700 \mathrm{~ms}$. The observation of desynchronization versus synchronization of alpha, even simultaneously during the immediate poststimulus time, depends on the method of power analysis (Klimesh et al., 2000): our method emphasizes the induced part, which includes the evoked alpha (contributing to the evoked potential, see Cacace and McFarland, 2003) and overrides the periand post-stimulus desynchronization. More interesting, however, was to notice that a small proportion of (evoked) alpha power was phase-locked to stimuli throughout the ISI, a phenomenon that we had observed previously in various different experiments, in spite of the short alpha wavelength as compared to the ISI. One may speculate that this part of the alpha generators could serve as a kind of task-time marker or estimator, and believe that competing hypotheses on alpha functional role can be reconciled: alpha synchronization may index cortical idling (Pfurtscheller, 2001), a concept that accounts for most of alpha behavior including the Berger effect and in drowsiness, but when occurring in the 'reference interval' in cyclical tasks such as the present, will also mean preparation for detection of forthcoming stimuli (Knyazev et al., 2006), around and immediately after which both (de-) and synchronization may be observed (Klimesh et al., 2000). Alpha scalp distribution included the expected posterior, occipital-parietal power maxima in all subjects, but with fine details in scalp distribution peculiar to each individual. However complex the individual map shapes, based on our own unpublished observations (for instance, one of the subjects presented an identical complex alpha-2 map during a visual verbal task performed 6 years before), we believe that alpha topography is fairly stable in the long term and possibly task-independent. This is in agreement with a few studies on stability of qEEG, although using fewer electrodes, that depict alpha as the most stable band within individuals (see Neuper et al., 2005). Our contribution, however, is the conclusion based on alpha analysis, but verified in all frequency bands, that the computation of corrected latency averages resulted in undistinguishable topography for the evoked or induced power bands. One very recent study using the standard 10-20 montage clearly corroborates this finding, for the alpha band (Hanslmayr et al., 2006).

The central finding of this work was the presence of beta band power increases throughout the ISI, peaking close to the S2 stimulus, in all subjects. Since the beta range is traditionally associated with wakefulness and behavioral arousal, we were interested in verifying the beta band generators, whether they would have a simple and common distribution across subjects. However, once we computed the topographic maps, we immediately noticed the qualitative similarity between induced beta and the SPs: common task-time behavior, multifocal, complex topography, highly variable across subjects, and significantly enhanced during the task as compared to the passive stimulation control condition. Thus, proceeding to compute beta burst averages centered in peaks occurring within the 700 to $1600 \mathrm{~ms}$ task time window, and model their generators by CDR, we obtained analogous results: Beta generators showed an equally complex pattern, comprising prefrontal and posterior cortical areas (as do SPs), highly variable across subjects, with only parietal area 7 demonstrating some, but variable, degree of relative current density in all subjects. Area 7 was also the only common SP generator region across subjects (Basile et al., 2006), a fact that may be attributed to the mere wakeful state, or that in other primates has been attributed to interested attention to the environment (Lynch et al., 1977; Yin and Mountcastle, 1978). We thus considered to 
have obtained a new index of attention, which could not have been observed by regular ERP averaging. The comparison between beta and SP generators by visual inspection (the reader may refer to the SP generator figure in Basile et al., 2006) revealed a largely complementary set of active areas between the two indexes: current foci were typically different from each other, forming mostly adjacent sets within subjects, and whenever there was an overlap of SP and beta generating areas, they did not correspond to the main generators in each case; that is, strong with weak in almost all cases. Moreover, SP generators were overall more spread over the cortical surface, and given the many cases of adjacent generator positions, it was unavoidable for us to speculate that SPs could represent a fringe effect stemming from the beta generating areas. SPs are for a long time known to be microscopically generated by a major contribution from the potassium buffering function of glia (Skinner and Molnar, 1983; Roitback, 1993; Mitzdorf, 1993), in situations of increased overall neural firing, as seems to occur areas active in the beta range. Finally, our phase analysis results present weak evidence that cortical areas active in the beta range also oscillate in phase synchrony with each other, at least roughly accompanying the power changes. However, the stability of partial averages (with respect to groups of electrodes ranked by peak latency), as well as independent studies using single-cell, extra-cellular recordings and model simulations offer stronger support to this view (Bibbig et al., 2002). This suggests that such areas become co-recruited, either reciprocally, or by some common subcortical projection(s).

A surprising and, if replicated, most important finding, regarded all frequency bands: their baseline or pre-S1 topographic distribution. In all cases and subjects, almost all of the task-induced power distribution was already manifested during baseline, and verified even during rest in three subjects. That is, individual-specific generators of each band (very similar, however, between delta, theta and beta ranges) are already active during resting wakefulness. This finding, also very recently observed in the alpha range by Hanslmayr and colleagues (2006), when combined with the indistinguishable topography between induced and evoked activity, and with the phase analysis results, may be interpreted into a general panorama regarding oscillatory activity: the generators of each sub-band are largely fixed in space and continuously active; for all bands, almost all of the task-induced power is accounted for by the same generators active during the baseline period. And during the task, combinations of three additional phenomena may occur, depending on the sub-band of oscillations: (1) increases and decreases in amplitude, that in some cases appear to be accompanied by roughly parallel (2) changes in coherence between varying proportions of the cell populations composing the generators, and (3) phaseresetting with respect to task-events. In the present study, for instance, it appears that delta and theta amplitude changes are least important, given that half of the subjects showed very low or no change in theta, or even decreases (2 subjects) in delta burst average amplitude. Alpha generators suffered resetting partly (that may be explained by being restricted to a proportion of cells within the same macroscopic areas), but most of their power increase was out of synchrony with task events. In the case of beta, the task-induced power increases was appear to be generated by synchronous areas, but out of phase with task events. The relative contribution by each of such phenomena thus depends on frequency band, and task, as concluded in other studies, some of which explicitly relate them to the composition of ERPs simultaneously obtained (Gruber et al., 2005; Hanslmayr et al., 2006; Valencia et al., 2006; and the interesting invasive study by Fell et al., 2004).

Taken together, our main findings lead us to the following implication to psychophysiology: as opposed to cortical activity linked to sensory stimulation (evoked potentials, delta, theta and partly alpha rhythms), which is simpler in distribution and more preserved across 
subjects, electrical activity directly related to expecting attention, namely the Slow Potential component of the ERP (corresponding to the $\mathrm{DC}-1 \mathrm{~Hz}$ induced bandpower) and the induced beta rhythm, is multifocal, complex in distribution, and highly variable across subjects. Moreover, it appears that when one engages in the task, it is largely the same individualspecific set of cortical areas, continuously active during simple resting wakefulness but without phase synchrony, that enter in phase and increase in power, and may recruit a few other, equally individualspecific areas. Our data, together with the results from the not many fMRI and PET studies that present individual data on event-related metabolic changes (Cohen et al., 1996; Herholz et al., 1996; Fink et al., 1997; Davis et al., 1998; Hudson, 2000; Brannen et al., 2001; Tzourio-Mazoyer et al., 2002), are compatible with the view of a "degenerate" mapping between presumed function and function implementing cortical areas (Noppeney et al., 2004). The main piece of knowledge that has guided our conventional hopes for a universal functional mapping are the fairly specific patterns of cortico-cortical connections in mammals (Pandya et al., 1988), especially so between visual cortices (Macko and Mishkin, 1985) but known to apply throughout the neocortex, including prefrontal areas (Pandya and Yeterian, 1990; Barbas, 1992). However, we believe that the mere complexity and number of possible cortico-cortical functional pathways are sufficient to allow the formation of variable sets of interconnected cortical areas across individuals, before and during execution of any given task. Therefore, we forecast the complete abandonment of the search for predetermined and unique functions to be attributed to given non-sensory-motor areas.

The implications of our results and conclusions to our own line of investigation are a reversal of focus: to aim exactly at understanding task-related and task-specific cortical activity, we will need to better comprehend the individual-specific cortical topography of the resting, baseline condition. Among critical aspects, we need to understand how the prevalently active sets of areas are formed during ontogeny and particularly during learning. We have only one subject as evidence for the stability in the order of one month, but three indicating a clear change in two or four years. It is fundamental to know how spontaneous is the formation of an individual resting pattern, as opposed to being subject to experimental or natural training influences. The possibility of directly influencing and expanding the resting pattern to apparently inactive areas would substantiate restorative neurology and neuropsychological procedures for a controlled reorganization of cortical circuits. On the other hand, if some cortical areas in one individual prove to be indeed 'silent', supposing that an exhaustive battery of experimental tasks can ever be implemented, such battery would become a procedure to complement the Wada and related tests to aid in neurosurgical planning. Finally, these overall conclusions are naturally compatible with the fact of high variability in clinical symptomatology after focal lesions: similar lesions in cortical 'association' areas lead to critical impairments in some individuals and may only be noticed in others by incidental laboratory examinations. Although we have recently used a method of scoring cortical activity by estimated cortical cytoarchitectonic area in each individual, previously to group or condition statistical comparisons (Basile et al., 2003), still searching for commonalities across subjects, equivalent attempts may be useful in the reconsideration of individual data from studies that have only presented group averages. We propose, as some other authors also explicitly do, that functional claims regarding cortical areas never be made based on group averaged data (Steinmetz and Seitz, 1991; Davis et al., 1998; Noppeney et al., 2004). The challenge of dealing with individual results, from the large number of studies already published, may at least help us to decide whether individual patterns are indeed arbitrary or follow rules, in which case new psychophysiological theories shall be developed. 


\section{ACKNOWLEDGMENTS}

This research was supported by the grants 03/02297-9 and 02/13633-7 from FAPESP, São Paulo, Brazil. We wish to thank Dr Cláudia Leite, Dr Edson Amaro Jr. and the staff from the Department of Radiology of the University of São Paulo Medical School, for kindly acquiring and preparing the MRI sets, and Márcio Costa for his valuable technical support.

\section{REFERENCES}

BARBAS H (1992) Architecture and cortical connections of the prefrontal cortex in the rhesus monkey. Advances in Neurology 57: 91-115

BASILE LFH, BALLESTER G, CASTRO CC and GATTAZ WF (2002) Multifocal slow potential generators revealed by high-resolution EEG and current density reconstruction. Int J Psychophysiol 45: 227-240

BASILE LFH, BALDO MV, CASTRO CC and GATTAZ WF (2003) The generators of slow potentials obtained during verbal, pictorial and spatial tasks Int $\mathbf{J}$ Psychophysiol 48: 55-65

BASILE LFH, YACUBIAN J, FERREIRA BLC, VALIM AC, and GATTAZ WF. Topographic abnormality of slow cortical potentials in schizophrenia. Brazilian Journal of Medical and Biological Research, 2004, 37: 97-109

BASILE LFH, BRUNETTI EP, PEREIRA JR JF, BALLESTER G, AMARO JR E, ANGHINAH R, RIBEIRO P, PIEDADE R, GATTAZ W F. Complex slow potential generators in a simplified attention paradigm. Int J Psychophysiology (in Press)

BIBBIG A, TRAUB RD, WHITTINGTON MA (2002) Long-range synchronization of gamma and beta oscillations and the plasticity of excitatory and inhibitory synapses: a network model. J Neurophysiol 88: $1634-54$

BRANNEN JH, BADIE B, MORITZ CH, QUIGLEY M, MEYERAND ME, HAUGHTON VM. (2001) Reliability of functional MR imaging with wordgeneration tasks for mapping Broca's area. AJNR Am J Neuroradiol 22: 1711-8

BRUNS A, ECKHORN R (2004) Task-related coupling from high- to low-frequency signals among visual cortical areas in human subdural recordings. Int $\mathrm{J}$ Psychophysiol 51: 97-116

BUCHNER H, KNOLL G, FUCHS M, RIENACKER A, BECKMAN R, WAGNER M, SILNY J, PESCH J (1997) Inverse localization of electric dipole current sources in finite element models of the human head. Electroenceph Clin Neurophysiol, 102: 267-278

COHEN MS, KOSSLYN SM, BREITER HC, DI GIROLAMO GJ, THOMPSON WL, ANDERSON AK, BROOKHEIMER SY, ROSEN BR, BELLIVEAU JW (1996) Changes in cortical activity during mental rotation. A mapping study using functional MRI. Brain, 119 (Pt 1): 89-100

DAVIS KD, KWAN CL, CRAWLEY AP, MIKULIS DJ (1998) Functional MRI study of thalamic and cortical activations evoked by cutaneous heat, cold, and tactile stimuli. J Neurophysiol 80: 1533-46
EDELMAN GM, GALLY JA (2001) Degeneracy and complexity in biological systems. Proc Natl Acad Sci USA 98: 13763-8

FELL J, DIETL T, GRUNWALD T, KURTHEN M, KLAVER P, TRAUTNER P, SCHALLER C, ELGER CE, FERNANDEZ G (2004) Neural bases of cognitive ERPs: more than phase reset. J Cogn Neurosci 16: 1595-604

FINK GR, FRACKOWIAK RS, PIETRZYK U, PASSINGHAM RE (1997) Multiple nonprimary motor areas in the human cortex. J Neurophysiol 77: 2164-74

FUCHS M, WAGNER M, WISCHMANN HA, KOHLER T, THEISSEN A, DRENCKHAHN R, BUCHNER H (1998) Improving source reconstructions by combining bioelectric and biomagnetic data. Electroencephalogr Clin Neurophysiol 107: 93-111

FUCHS M, WAGNER M, KOHLER T, WISCHMANN HA (1999) Linear and nonlinear current density reconstructions. J Clin Neurophysiol 16: 267-95

GRUBER WR, KLIMESCH W, SAUSENG P, DOPPELMAYR M (2005) Alpha phase synchronization predicts $\mathrm{P} 1$ and $\mathrm{N} 1$ latency and amplitude size. Cereb Cortex 15: 371-7

HANSLMAYR S, KLIMESCH W, SAUSENG P, GRUBER W, DOPPELMAYR M, FREUNBERGER R, PECHERSTORFER T, BIRBAUMER N (2006) Alpha Phase Reset Contributes to the Generation of ERPs. Cereb Cortex. (in Press)

HERHOLZ K, THIEL A, WIENHARD K, PIETRZYK U, VON STOCKHAUSEN HM, KARBE H, KESSLER J, BRUCKBAUER T, HALBER M, HEISS WD (1996) Individual functional anatomy of verb generation. Neuroimage 3: 185-94

HUDSON AJ. Pain perception and response: central nervous system mechanisms (2000) Can J Neurol Sci 27: $2-16$

HYVARINEN A, OJA E (2000) Independent component analysis: algorithms and applications. Neural Netw 13: 411-30

LACHAUX JP, RODRIGUEZ E, MARTINERIE J, VARELA FJ (1999) Measuring phase synchrony in brain signals. Hum Brain Mapp 8: 194-208

LYNCH JC, MOUNTCASTLE VB, TALBOT WH, YIN TC (1977) Parietal lobe mechanisms for directed visual attention J Neurophysiol 40: 362-89

MACKO KA, MISHKIN M (1985) Metabolic mapping of higher-order visual areas in the monkey. Res Publ Assoc. Res Nerv Ment Dis 63: 73-86

MCCALLUM WC (1988) Potentials related to expectancy, preparation and motor activity. In: Handbook of Electroencephalography and Clinical Neurophysiology. Human Event-Related Potentials (revised series vol. 3) TW Picton (Ed.). Elsevier Science Publishers, 427-534

MITZDORF U (1993). Contributions of extracellular potassium increases to transient field potentials (review of data). In: Slow Potential Changes in the Brain. Chap 16. Haschke W, Roitbak AI, Speckmann, EJ (Eds.). Birkhäuser. Boston

NOPPENEY U, FRISTON KJ and CATHY J. (2004) Price Degenerate neuronal systems sustaining cognitive functions Journal of Anatomy 205: 433-442

PANDYA DN, YETERIAN EH (1990) Prefrontal cortex in relation to other cortical areas in rhesus monkey: Arquitecture and connections. In: Progress in Brain Research, vol.85. Uylings HBM, Van Eden CG, De Bruin JPC, Corner MA, Feenstra, MGP (Eds.). Elsevier Science Publishers BV. 63-94

PANDYA DN, SELTZER, B. AND BARBAS, H (1988) Input-output organization of the primate cerebral cortex. Comparative primate biology, 4: 39-80 
POSNER MI (1980) Orienting of attention. Q J Exp Psychol 32: 3-25

POSNER MI, SNYDER CR, DAVIDSON BJ (1980) Attention and the detection of signals. J Exp Psychol 109: $160-74$

RAMIREZ PM, DESANTIS D, OPLER LA (2001) EEG biofeedback treatment of ADD. A viable alternative to traditional medical intervention? Ann N Y Acad Sci 931: $342-58$

ROITBAK AI (1993). Cortical slow potentials, depolarization of glial cells, and extracellular potassium concentration. In: Slow Potential Changes in the Brain. Chap 14. Haschke W, Roitbak AI, Speckmann, E J. (Eds.). Birkhäuser. Boston

SKINNER JE, MOLNAR M (1983). Event-related extracellular potassium ion activity changes in frontal cortex of the conscious cat. J Neurophysiol 49: 204-215

Steinmetz H, Seitz RJ (1991) Functional anatomy of language processing: neuroimaging and the problem of individual variability Neuropsychologia 29: 1149-61

TALAIRACH J, TOURNOUX P (1993). Referentially oriented cerebral MRI anatomy. Atlas of stereotaxic anatomical correlations for gray and white matter. Thieme Medical Publishers, Inc. New York

TALAIRACH J, TOURNOUX P (1997). Co-planar stereotaxic atlas of the human brain. Thieme Medical Publishers, Inc. New York

TALLON-BAUDRY C, BERTRAND O, DELPUECH C, PERNIER J (1996) Stimulus specificity of phase- locked and non-phase-locked $40 \mathrm{~Hz}$ visual responses in human. J Neurosci 16: 4240-9

TZOURIO-MAZOYER N, JOSSE G, CRIVELLO F, MAZOYER B (2002) Interindividual variability in the hemispheric organization for speech. Neuroimage 16: 765-80

UYLINGS HB, RAJKOWSKA G, SANZ-ARIGITA E, AMUNTS K, ZILLES K (2005) Consequences of large interindividual variability for human brain atlases: converging macroscopical imaging and microscopical neuroanatomy. Anat Embryol (Berl) 210: 423-31

VALENCIA M, ALEGRE M, IRIARTE J, ARTIEDA J (2006) High frequency oscillations in the somatosensory evoked potentials (SSEP's) are mainly due to phase-resetting phenomena. J Neurosci Methods 154: $142-148$

VANDENBROUCKE MW, GOEKOOP R, DUSCHEK EJ, NETELENBOS JC, KUIJER JP, BARKHOF F, SCHELTENS P, ROMBOUTS SA (2004) Interindividual differences of medial temporal lobe activation during encoding in an elderly population studied by fMRI. Neuroimage 21: 173-80

WHELESS JW, CASTILLO E, MAGGIO V, KIM HL, BREIER JI, SIMOS PG, PAPANICOLAOU AC (2004) Magnetoencephalography (MEG) and magnetic source imaging (MSI). Neurologist 10: 138-53

YIN TC, MOUNTCASTLE VB (1978) Mechanisms of neural integration in the parietal lobe for visual attention. Fed Proc 37: 2251-7 